\title{
Neural Predictive Control of Broiler Chicken Growth
}

\author{
T.G.M. Demmers ${ }^{*}$ Y. Cao ${ }^{* *}$ S. Gauss ${ }^{* * *}$ J.C. Lowe ${ }^{* * * *}$ \\ D.J. Parsons ${ }^{\dagger}$ C.M. Wathes ${ }^{\ddagger}$ \\ * The Royal Veterinary College, UK (e-mail: tdemmers@rvc.ac.uk). \\ ** Cranfield University, UK (e-mail: y.cao@cranfield.ac.uk). \\ *** The Royal Veterinary College, UK (e-mail: sgauss@rvc.ac.uk). \\ **** The Royal Veterinary College, UK (e-mail: jlowe@rvc.ac.uk). \\ $\dagger$ Cranfield University, UK (e-mail: d.parsons@cranfield.ac.uk). \\ $\ddagger$ The Royal Veterinary College, UK (e-mail: cmwathes@rvc.ac.uk).
}

\begin{abstract}
Active control of the growth of broiler chickens has potential benefits for farmers in terms of improved production efficiency, as well as for animal welfare in terms of improved leg health. In this work, a differential recurrent neural network (DRNN) was identified from experimental data to represent broiler chicken growth using a recently developed nonlinear system identification algorithm. The DRNN model was then used as the internal model for nonlinear model predicative control (NMPC) to achieve a group of desired growth curves. The experimental results demonstrated that the DRNN model captured the underlying dynamics of the broiler growth process reasonably well. The DRNN based NMPC was able to specify feed intakes in real time so that the broiler weights accurately followed the desired growth curves ranging from $-12 \%$ to $+12 \%$ of the standard curve. The overall mean relative error between the desired and achieved broiler weight was $1.8 \%$ for the period from day 12 to day 51 .
\end{abstract}

Keywords: Predictive Control, Broiler, Growth, Optimal Control, System Identification, Neural Network Models.

\section{INTRODUCTION}

This work forms part of a programme to determine, model and control the biological and physical responses and interactions of poultry to dynamic changes in their physical environment. In particular, it studies the growth and behaviour of broiler chickens reared for meat production and their ammonia emissions in response to dynamic changes in feed quantity, light intensity and relative humidity. This paper focusses primarily on growth.

Growth of an animal integrates various physiological and environmental processes, so weight gain is not only a valuable measure of economic performance, but also a convenient measure of environmental response. Maximal growth rate as a function of feed intake is the most important parameter from the perspective of growers, because feed is the biggest cost in the production of broilers. Recently other physiological processes such as skeletal development and activity have also been considered. Slower growth in the early stages of broiler development reduces the incidence of lameness, the most important animal welfare issue in broiler production [Butterworth and Arnould, 2009].

Frost et al. [1997] argued that livestock production systems contain multiple interconnected processes that need to be managed to meet several performance criteria, including economic, animal welfare and environmental targets. Traditional management was, and still is, largely based on experience and is not good at integrating processes and performance criteria. An example is the use of climate (temperature) controllers. Development of the climate controller was through observing animal performance and behaviour [Charles and Walker, 2002]. However, control was through temperature measurement alone, discarding any information from the animal. The stockman still had to intervene if the response of the animals indicated that the temperature control was imperfect. The proposed solution was to move towards integrated closed-loop, model-based control systems, by first developing controllers for the key processes, using sensor technology capable of measuring animal responses, that was becoming available.

The nutritional and environmental requirements of broilers are well understood [Gous et al., 1999], which has enabled the development of mechanistic models to predict broiler growth from feed inputs [Emmans, 1995]. These models and the science underlying them have been used to create plans for nutrition and weight gain [Aviagen, 2002]. However, the dynamic responses of animals to (sudden) changes in the environment are less well understood and fewer models exist. Furthermore, Wathes et al. [2008] states that in general mechanistic models are not suitable for control purposes, because they are often overly complex, with too many parameters, although these have biological meanings, and inaccurate, since parameter values may change over time and space.

Recently, data-based models describing the response of the growing broiler to changes in feed quantity have been explored as an alternative to mechanistic models. Data- 
based modelling techniques estimate the unknown model parameters of any abstract mathematical model structure from measurements of process inputs and outputs. In principle, the parameters can be estimated on-line resulting in an adaptive model that can cope with the characteristics of most biological processes, i.e. individual, time variant and dynamic [Aerts et al., 2003b]. This type of model has the advantage that no a priori knowledge of the process is required, although the latter is beneficial whilst developing the model. However, in contrast to mechanistic models, the parameters have no biological meaning. The resulting model will in general be more compact and therefore suitable for control purposes. As a result data-based models are widely used for process control in other industries. Various approaches to modelling broiler growth have been used, including hyperbolastic models [Ahmadi and Mottaghitalab, 2007], artificial neural networks [Ahmadi and Mottaghitalab, 2008] and recursive linear models [Aerts et al., 2003b].

Frost et al. [2003] and Stacey et al. [2004] described the development of a system based on a mechanistic model to control the feeding of broiler chickens to achieve a given time-weight performance. The system was developed on farm scale (over 30,000 birds/house) using a feeding system where the diet composition was controlled by blending two different feeds and growth was monitored by perch weighers. It aimed to optimise the feed blend to minimise the errors from a planned growth curve from the current day to slaughter, and was able to deliver birds of the correct weight, except when growth was inhibited by disease. The use of a simulation model required the specification of several genotype-dependent parameters and feed analysis in terms of several nutrients.

For the reasons discussed above, a data-based approach was followed on laboratory scale by Aerts et al. [2003a] and at a larger scale by Cangar et al. [2008], in which the quantity of feed presented was controlled using model predictive control. They used a recursive linear model with time varying parameters to predict weight 3-7 days ahead [Aerts et al., 2003b]. Using online prediction of the feed quantity, control of broiler growth along a target trajectory proved possible within certain boundary conditions. Most notably, the period during which growth could be restricted without affecting the ability of the broiler to reach the target weight was limited to the early stages of growth (age 7-30 days). Growing broilers to the required target weight using online control resulted in a mean relative error of $6-10 \%$ in live weight.

The method described here shares some of the characteristics of the above approaches and aims to overcome some of their limitations. The model is empirical, so does not require genetic parameters or detailed feed analyses, but simulates growth from hatching to slaughter. Based on this model, the controller is designed to optimise feeding over the complete period of growth instead of a fixed horizon. The control strategy aims to optimise the system by reducing the feed intake to save cost, minimising the deviation of bird weight from a predefined grow curve to ensure the final target is smoothly achieved and at the same time restricting the daily change in the intake to avoid potential stress on the birds. These objectives are combined into a single cost function as a weighted sum of these criteria.

The paper is organised as follows. In section 2, after a brief description of broiler growth and the experimental data, the DRNN model is introduced and developed to represent the growth dynamics. The growth control problem is then defined in section 3 and solved using the DRNN model and the NMPC framework. The performance of the DRNN model and the NMPC algorithm are demonstrated through experiments in section 4. A discussion of the results and the conclusions are given in section 5 .

\section{WEIGHT-FEED MODEL IDENTIFICATION}

Growth of any organism is a complicated nonlinear dynamic process, which is difficult to model from first principles. Most conventional system identification approaches use linear model structures, such as the autoregressive moving average with exogenous input model (ARMAX), which are not capable of capturing the nonlinearity covering the entire growth process of broilers. Due to their ability to approximate any nonlinear function, recurrent neural networks (RNN) are widely used for nonlinear system identification. However, most available RNN models are in discrete time, which can only work for the specific sampling rate with which the model is trained. In order to develop a dynamic model to control the entire growth process with potentially variable sampling rate, the differential RNN (DRNN) and the associated automatic differentiation based training algorithm developed by AlSeyab and Cao [2008b,a] were adopted for this work.

A first order DRNN model with two hidden nodes represented as follows is adopted to represented the boiler growth process.

$$
\dot{x}=w_{5} \sigma\left(w_{1} x+w_{3} u\right)+w_{6} \sigma\left(w_{2} x+w_{4} u\right)
$$

where $x$ and $u$ are the weight and feed intake for a single bird, respectively, $\sigma(x)=\frac{e^{x}-e^{-1}}{e^{x}+e^{-1}}$ and $w_{1}, \ldots, w_{6}$ are model parameters to be determined. The model structure is determined based on the intuitive assumption that from any initial weight, $x_{0}$, if the feed intake is zero, then the boiler weight will gradually decay to zero.

To generate data for training and validating the model, broilers were grown from 1 day old to 51 days and exposed to dynamic (sudden) changes in the inputs, feed amount, light intensity and relative humidity $(\mathrm{RH})$, from day 12 onwards. To ensure a measurable response in output, the change in the input was set unrealistically large compared to normal broiler production practise. Feed amount was set at either $90 \%$ or $110 \%$ of normal rates. Light intensity was set at either 10 or 100 lux and RH at $56 \%$ or $70 \%$. The frequency of change was set according to the time required to reach a new steady state in the output, i.e. hours for the light intensity and 3-7 days for feed amount and RH.

A two-level (change or no change) of three-factor (feed amount, light intensity and $\mathrm{RH}$ ) factorial design requiring $2^{3}=8$ identical rooms was used and repeated in three trials. Each possible combination of inputs was randomly allocated to a room in each of the three trials. This experimental design potentially allowed identification of 
interactions between the processes: growth, activity and ammonia emission, affected by feed amount, light intensity and $\mathrm{RH}$, respectively.

Each room housed 262 broilers (Ross 308) on a bed of woodshavings. Stocking density was $33 \mathrm{~kg} \mathrm{~m}^{-3}$. The bird weight was measured continuously using a weighing platform suspended from a load cell (Fancom 747 series bird weight platform and computer). Specially formulated broiler feeds were weighed and dosed automatically to each room (Fancom 771 feed computer) four times a day. Feed quantity dosed and bird weights in each room were recorded automatically four times per day from day 3-51. Other environmental variables, such as temperature, $\mathrm{RH}$ and light intensity, were monitored and recorded at least four times per day.

To determine the six model parameters, experimental data from the trails described above were used. Each batch contained the input and output data for one room from one trial. The training data set consisted of six batches, two from each trial. Another six batches were selected for validation.

The training process started from a set of randomly generated parameters. The growth of a batch was then calculated from the initial weight and the feed intakes recorded in the data by solving the model equation (1) using the automatic differentiation approach described by Cao [2005]. Let the bird weight recorded in experiments and estimated from (1) at each sampling time be $x_{k}$ and $\hat{x}_{k}, k=1, \ldots, N$, respectively. Then the training process aimed to minimise the following cost function by adjusting the model parameters $w_{1}, \ldots, w_{6}$

$$
\min _{w_{1}, \ldots, w_{6}} \sum_{k=1}^{N}\left(x_{k}-\hat{x}_{k}\right)^{2}+\sum_{k}^{6} \alpha w_{k}^{2}
$$

where $\alpha$ is a weighting factor for the model parameters. The second term of the cost function is for rigid regulation, which improves the model generality.

The optimization in (2) was converted into a standard nonlinear least squares problem and solved using the Levenberg-Marquardt (LM) algorithm (see [Marquardt, 1963]), where the model parameters were iteratively updated to reduce the cost function until the algorithm converged or the validation cost started to increase. To avoid the training process being trapped in a local minimum, the optimization procedure was repeated with different sets of randomly generated initial parameters until a satisfactory model was obtained. The final model parameters obtained were:

$$
\begin{aligned}
& w_{1}=-2.8456 \times 10^{-4} \\
& w_{3}=-2.5539 \times 10^{-3} \\
& w_{5}=756.5
\end{aligned}
$$

$$
\begin{aligned}
& w_{2}=1.0162 \times 10^{-4} \\
& w_{4}=4.2284 \times 10^{-3} \\
& w_{6}=1488.5
\end{aligned}
$$

This system is stable at the equilibrium point $x=0$ and $u=0$. This can be verified by the pole of the system at this point, $p=w_{1} w_{5}+w_{2} w_{6}=-0.064<0$. Therefore, the model indicates that for zero intake, the weight of a bird will eventually decay to zero.

Typical performance of the trained DRNN model is represented for one of the remaining 12 test batches in Figure 1, which shows that the trained DRNN was able to predict the bird weight satisfactorily even when the actual feed intake was modulated by regular step changes.

Trail 4, Room 6, $\mathrm{mse}=7.14269 \mathrm{e}-005, \mathrm{std}=0.000241294$
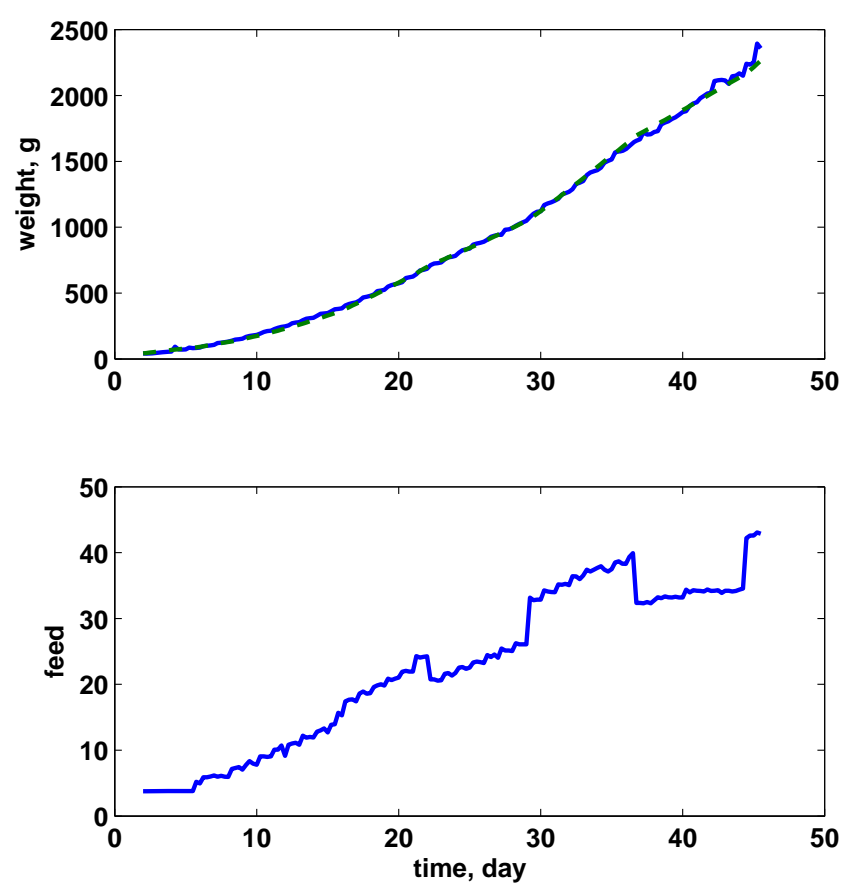

Fig. 1. DRNN model testing. Top: the actual (solid-line) and predicted (dashed-line) broiler weight; Bottom: the actual feed intake.

\section{BROILER GROWTH CONTROL}

In theory, using the identified DRNN model, many optimal control problems can be investigated, such as minimum time control, where feed intakes are calculated such that birds can grow as fast as possible to reach the target weight, and the minimum food problem, where optimal feed intake is designed such that the total food consumption is minimized to achieve the same target weight on the target day. However, due to the limited experimental data, upon which the model was based, it would not be applicable to some extreme situations, such as very low and high feed intakes. To ensure the model was working within a reliable range that would not compromise broiler welfare, a regulation control problem was constructed to design optimal feed intake such that the actual bird growth followed a predesigned curve smoothly with the minimum feed intake.

The above regulation problem was solved through a nonlinear model predictive control (NMPC) scheme. In the $\mathrm{NMPC}$, at each sampling point, $t_{0}$, the average weight of a bird predicted by the model, $x_{0}$ is compared with the measured weight, $x_{m}$. The difference, $n=x_{m}-x_{0}$ is treated as the disturbance. This disturbance is assumed to be constant within the prediction horizon, $t_{0} \leq t \leq t_{f}$. Therefore, to correct the error caused by this disturbance, the actual set-point at a time point, $t$, within the prediction horizon is biased as $\hat{x}(t)=x_{r}(t)+n$, where $x_{r}(t)$ is the target weight. Then, the optimal control problem to be solved at each sampling point, $t_{0}$ is stated as follows. 


$$
\begin{aligned}
\min _{u} & \sum_{t=t_{0}}^{t_{f}}\left[\alpha_{1}^{2}(x(t)-\hat{x}(t))^{2}+\alpha_{2}^{2} v^{2}(t)+\alpha_{3}^{2}(\Delta v(t))^{2}\right] \\
\text { s.t. } & \dot{x}=w_{5} \sigma\left(w_{1} x+w_{3} u\right)+w_{6} \sigma\left(w_{2} x+w_{4} u\right) \\
& x\left(t_{0}\right)=x_{0} \\
& x\left(t_{f}\right)=x_{f}
\end{aligned}
$$

where, $v^{2}(t)=u(t)$ is the feed intake at day $t, \Delta v(t)=$ $v(t)-v(t-1), t_{0}$ and $t_{f}$ are current and final days, respectively, $x_{0}$ and $x_{f}$ are current and final weights, respectively, $\alpha_{1}, \alpha_{2}$ and $\alpha_{3}$ are weights of the optimization problem for weight accuracy, food consumption and smoothness respectively. Note that although the optimal control problem in (3) is open loop, the correction of modelling error, $\hat{x}(t)=x_{r}(t)+x_{m}\left(t_{0}\right)-x_{0}$ uses the real measured weight, $x_{m}\left(t_{0}\right)$, hence the actual control is feedback control.

The problem can be cast as a standard nonlinear least square problem, $\min _{\mathbf{u}} \mathbf{e}^{T} \mathbf{e}$, with residuals, e defined as follows.

$$
\mathbf{e}=\left[\begin{array}{c}
\alpha_{1}\left(x\left(t_{0}+1\right)-\hat{x}\left(t_{0}+1\right)\right) \\
\vdots \\
\alpha_{1}\left(x\left(t_{f}\right)-\hat{x}\left(t_{f}\right)\right) \\
\alpha_{2} v\left(t_{0}\right) \\
\vdots \\
\alpha_{2} v\left(t_{f}-1\right) \\
\alpha_{3} \Delta v\left(t_{0}\right) \\
\vdots \\
\alpha_{3} \Delta v\left(t_{f}-1\right)
\end{array}\right]
$$

The corresponding Jacobian, $\mathbf{J}=\partial \mathbf{e} / \partial \mathbf{u}$ can be derived through automatic differentiation as explained by AlSeyab and Cao [2008b]. The optimal values of $\mathbf{v}=$ $\left[v\left(t_{0}\right) \cdots v\left(t_{f}-1\right)\right]^{T}$ are then obtained iteratively using the LM algorithm [Marquardt, 1963]:

$$
\mathbf{v}_{k+1}=\left(\mathbf{J}_{k}^{T} \mathbf{J}_{k}+\mu \mathbf{I}\right)^{-1} \mathbf{J}_{k}^{T} \mathbf{e}_{k}
$$

where $\mathbf{e}_{k}$ and $\mathbf{J}_{k}$ are the residuals and the Jacobian corresponding to $\mathbf{v}_{k}, \mu$ is a parameter adjusted by the algorithm to maintain a fast convergence.

Once the iteration has converged, the first instance of the obtained optimal solution, $\mathbf{v}$ is converted into the feed intake, $u\left(t_{0}\right)=v^{2}\left(t_{0}\right)$ and applied to the real system. The whole procedure will be repeated at next sampling time when a new measured average bird weight, $x_{m}$ is available.

\section{VALIDATION OF THE GROWTH CONTROL ALGORITHM}

To validate the control algorithm developed in the previous section, fresh experiments were designed and carried out. In these experiments, four growth curves were devised for the controller to attempt to follow as closely as possible by predicting the required feed intake. These new growth curves were derived from the standard growth curve provided by Aviagen [2002], i.e. reaching a weight of $2.85 \mathrm{~kg}$ at 50 days of age, and were used for the development of the controller. Up to day 12 the broilers were grown according to the standard curve, then, from day $12-50$, followed the new growth curves. The four new growth curves were specified as
- standard curve

- $+12 \%$ of standard curve

- $-12 \%$ of standard curve

- $-12 \%$ to day 30 followed by $+12 \%$ of standard curve (slow growth followed by recovery growth)

The broiler growth controller was tested using four of the eight available rooms. Each growth curve was tested with one room. Each room was initially stocked with 265 day-old chicks (Ross 308). Environmental conditions were kept identical to the conditions used in the training and model validation trials, apart from the frequency of light intensity change and number of meals fed daily. The total daily intake of each room was set by the controller. The controller was used for on-line calculation of the feed intake, however with a 24-hour delay in implementation of the calculated feed intake through a manual adjustment of the feed dosed.

The initial results from the first 4 batches are summarised in Table 1, where the four controlled (actual) weights at day 50 are compared with their corresponding target values taken from the prescribed growth curves. The predicted total feed intake was calculated from the sum of the controller-predicted feed dosage rate. The actual total feed intake was calculated from the sum of the feed dosed per room for day 12-49, corrected for the actual number of birds present. The mean relative error and maximum deviation of the actual weights from day $12-50$ were calculated as percentages, where the mean relative error, $\bar{\varepsilon}$ and the maximum deviation, $\sigma_{\max }$ are defined based on the actual weight, $w_{\text {act }}$ and the corresponding theoretical weight, $w_{\text {th }}$ as follows.

$$
\begin{array}{r}
\bar{\varepsilon}=\frac{1}{39} \sum_{d=12}^{50}\left|\frac{w_{\mathrm{act}}(d)-w_{\mathrm{th}}(d)}{w_{\mathrm{th}}(d)}\right| \\
\sigma_{\max }=\max _{12 \leq d \leq 50}\left|\frac{w_{\mathrm{act}}(d)-w_{\mathrm{th}}(d)}{w_{\mathrm{th}}(d)}\right|
\end{array}
$$

Daily comparisons of controlled against modelled and standard growth curves are shown in Figures 2 to 5 for the standard growth curve and $+12 \%,-12 \%$ and $-12 \%$ followed by $+12 \%$ of standard growth curves, respectively.

\section{Standard growth curve (Aviagen)}

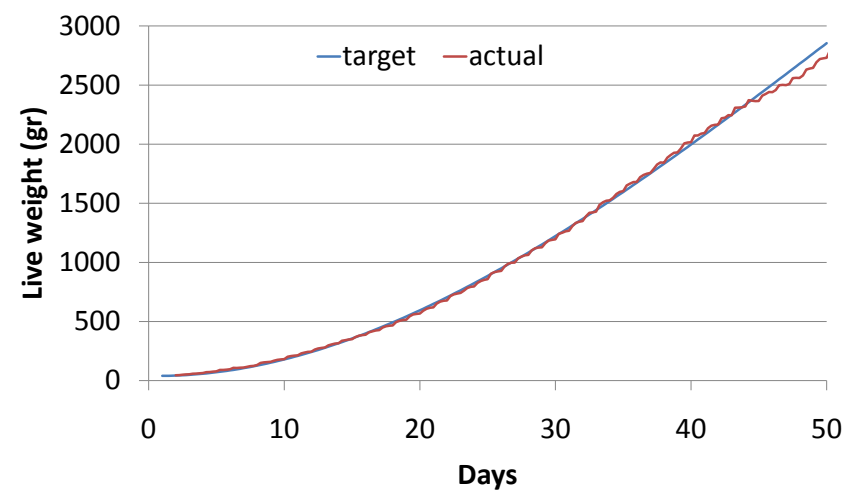

Fig. 2. Theoretical standard and actual achieved growth curves. The standard growth curve (Aviagen) is plotted for comparison. 
Table 1. Theoretical live weight and achieved live weight of the broilers at age 50 days and goodness of fit of the achieved live weight compared to the set growth curve from day $12-50$. Predicted and actual total feed intake per bird and feed conversion ratio (FCR) for the period of day $12-49$.

\begin{tabular}{lccccc}
\hline \hline Growth curve & unit & $\begin{array}{c}\text { Standard } \\
\text { (Aviagen) }\end{array}$ & $\begin{array}{c}+12 \% \text { of } \\
\text { standard }\end{array}$ & $\begin{array}{c}-12 \% \text { of } \\
\text { standard }\end{array}$ & $\begin{array}{l}-12 \% \&+12 \% \\
\text { of standard }\end{array}$ \\
\hline Theoretical & $\mathrm{kg}$ & 2.85 & 3.20 & 2.51 & 2.85 \\
Actual & $\mathrm{kg}$ & 2.73 & 3.10 & 2.44 & 2.72 \\
Mean relative error & $\%$ & 1.8 & 1.8 & 2.8 & 1.6 \\
Maximum deviation & $\%$ & 5.2 & 6.0 & 16.3 & 5.0 \\
\hline \multicolumn{7}{c}{ Total feed intake from day $12-49$} \\
\hline Predicted & $\mathrm{kg}$.bird ${ }^{-1}$ & 4.66 & 4.99 & 4.30 & 4.62 \\
Actual & $\mathrm{kg} \cdot$ bird $^{-1}$ & 4.59 & 5.04 & 4.31 & 4.62 \\
Feed conversion Ratio & - & 1.91 & 1.84 & 2.02 & 1.93 \\
\hline
\end{tabular}

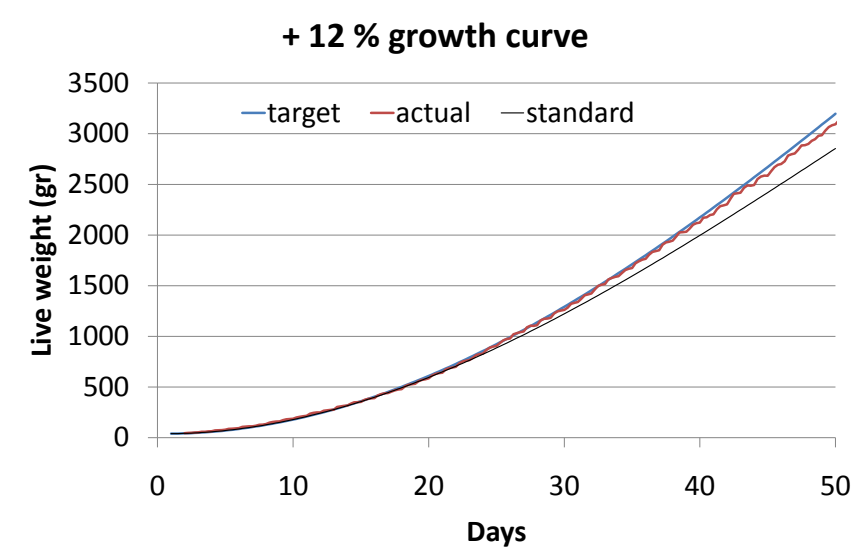

Fig. 3. Theoretical $+12 \%$ of standard and actual achieved growth curves. The standard growth curve (Aviagen) is plotted for comparison.

\section{$-12 \%$ growth curve}

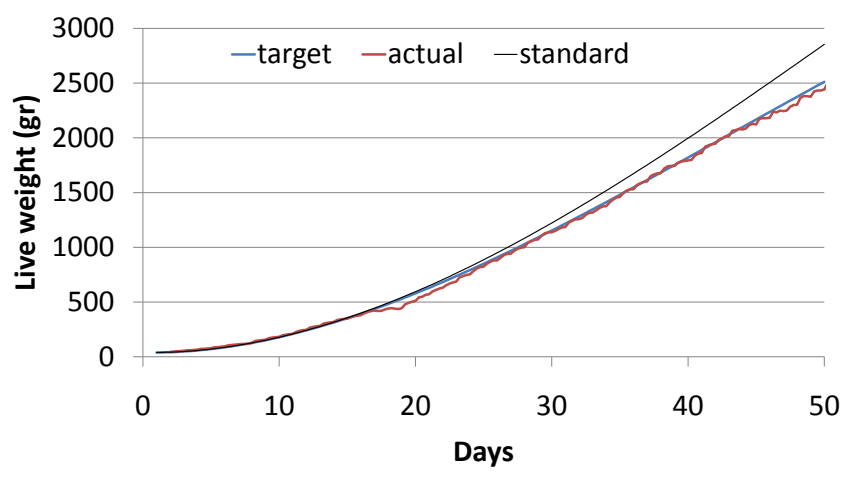

Fig. 4. Theoretical $-12 \%$ of standard and actual achieved growth curves. The standard growth curve (Aviagen) is plotted for comparison.

The results clearly indicate that the controller is capable of predicting the feed intake required to reach the end weight and follow the reference growth curves well with an mean relative error less than $2 \%$, except for the $-12 \%$ curve. The larger mean relative error in the $-12 \%$ growth curve was caused by a malfunction in the feeding equipment from day 16-19 causing the birds to be fed the correct feed amount, but at very irregular intervals, potentially inhibiting growth (maximum deviation from curve was $-16 \%$ ). However, the controller was able to return the
- $12 \&+12 \%$ growth curve

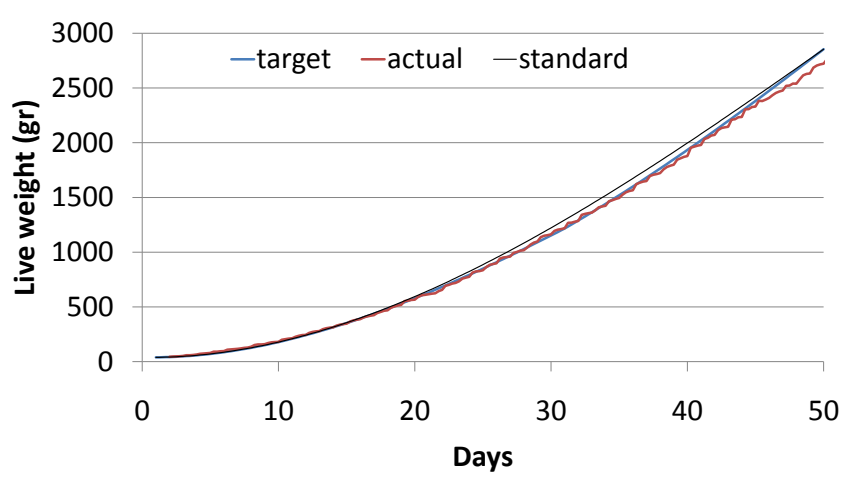

Fig. 5. Theoretical $-12 \%$ followed by $+12 \%$ of standard and actual achieved growth curves. The standard growth curve (Aviagen) is plotted for comparison.

growth to the set curve within 4 days. Excluding this period reduced the mean relative error to $1.9 \%$. Overall the mean relative error in this work is much lower than the $7-9 \%$ reported by Cangar et al. [2008]. The authors suggested that this high error might be largely due to different conditions and systems for the weighing and feed delivery used for generating data for creating and validating the model (small scale, "ideal" conditions) and for the validation of the control algorithm. In our work all steps were done on the same scale, same conditions and with the same equipment.

For all four growth curves, the projected end weight was met within small tolerances. From day 42 onwards the actual bird weight started to deviate from the theoretical bird weight (slower growth). This could be a undesirable feature of the DRNN model used. However, it also coincided with the introduction of the withdrawal grower diet which in theory differs in composition from the normal grower diet in the absence of coccidiostats only. The absence of the coccidiostats should not affect the growth or feed conversion, but it is not evident from the feed analysis if other minor changes were made to the feed composition between the two deliveries that could have affected the growth. In contrast to findings by Cangar et al. [2008] in these trials the Ross 308 bird appeared to be capable of recovery growth (see Figure 5), i.e. the broilers were capable of regaining weight in excess of equivalent growth by the standard growth curve beyond 31 days. One reason 
for this difference is the lower energy and protein content of the diets used in this work compared with current industry standards (approximately 15\% lower). The standard growth curve used was also set below the maximum potential growth curve given by Aviagen [2002]. Hence, the broilers were capable of utilising the additional protein and energy provided as the maximum growth potential had not yet been reached.

The DRNN model used in the controller controlled not only the daily feed intake on line, but predicted accurately the required feed intake for the whole of the growing period. This novel addition will be very useful to farmers when deciding on a growth curve suitable for various scenarios. From the four growth curves used in this trial the $+12 \%$ of standard growth curve is better from an economic point of view, as it has by far the lowest feed conversion ratio (FCR). Using the slow growth with recovery growth option, has potential advantages for animal welfare in terms of leg health and proved to be no worse in achieving the final weight with a similar FCR and total feed intake requirement.

\section{CONCLUSIONS}

An accurate differential recurrent neural network model of broiler growth has been identified, validated and tested successfully. The DRNN model accurately described the dynamic time variable growth of broilers. Typically the mean square error and standard deviation between the model and data were of the order of 0.02 and 0.03 , respectively.

The nonlinear model predictive controller, incorporating the DRNN model, was constructed to predict the feed quantity required for the broilers to grow following predetermined growth curves. Preliminary results showed that the NMPC accurately predicted the feed quantity to achieve a range of predetermined growth curves. The mean relative error for the period from day $12-50$ was $1.8 \%$. The NMPC was capable of accurately predicting compensatory growth rates following two days of retarded growth rates due to feeding equipment failure. In addition, the controller was able to predict the total feed intake for the whole growth period accurately.

\section{ACKNOWLEDGEMENTS}

This work was funded by the Biotechnology and Biological Sciences Research Council. The authors would like to thank the staff of the Biological Services Unit for providing excellent stockmen during the trials.

\section{REFERENCES}

J.M. Aerts, S. Van Buggenhout, E. Vranken, M. Lippens, J. Buyse, E. Decuypere, and D. Berckmans. Active control of the growth trajectory of broiler chickens based on online animal responses. Poultry Science, 82(12): 1853-1862, 2003a.

J.M. Aerts, M. Lippens, G. De Groote, J. Buyse, E. Decuypere, E. Vranken, and D. Berckmans. Recursive prediction of broiler growth response to feed intake by using a time-variant parameter estimation method. Poultry Science, 82(1):40-49, 2003 b.
H. Ahmadi and M. Mottaghitalab. Hyperbolastic models as a new powerful tool to describe broiler growth kinetics. Poultry Science, 86:2461-2465, 2007.

H. Ahmadi and M. Mottaghitalab. Predicting performance of broiler chickens from dietary nutrients using group method of data handling-type neural networks. British Poultry Science, 49(3):315-320, 2008.

R.K. Al-Seyab and Y. Cao. Differential recurrent neural network based predictive control. Computers and Chemical Engineering, 32(7):1533-1545, 2008a.

R.K. Al-Seyab and Y. Cao. Nonlinear system identification for predictive control using continuous time recurrent neural networks and automatic differentiation. Journal of Process Control, 18(6):568-581, 2008b.

Aviagen. Broiler management manual. Aviagen Ltd, Newbridge, Midlothian, Scotland, UK., 2002.

A. Butterworth and D. Arnould. Standardisation of measures of broiler lameness. In B. Forkman and L. Keeling, editors, Assessment of Animal Welfare Measures for Layers and Broilers., number 9 in Welfare Quality Reports, pages 31-39, Cardiff, UK, 2009. Cardiff University.

O. Cangar, J.-M. Aerts, E. Vranken, and D. Berckmans. Effects of different target trajectories on the broiler performance in growth control. Poultry Science, 87(11): 2196-2207, 2008.

Y. Cao. A formulation of nonlinear model predictive control using automatic differentiation. Journal of Process Control, 15(8):851-858, 2005.

D.R. Charles and A.W. Walker. Poultry Environment Problems. A guide to solutions. Nottingham University Press, Nottingham, UK., 2002.

G.C. Emmans. Problems in modeling the growth of poultry. Worlds Poultry Science Journal, 51(1):77-89, 1995.

A.R. Frost, C.P. Schofield, S.A. Beaulah, T.T. Mottram, J.A. Lines, and C.M. Wathes. A review of livestock monitoring and the need for integrated systems. Computers and Electronics in Agriculture, 17(2):139-159, 1997.

A.R. Frost, D.J. Parsons, K.F. Stacey, A.P. Robertson, S.K. Welch, D. Filmer, and A. Fothergill. Progress towards the development of an integrated management system for broiler chicken production. Computers and Electronics in Agriculture, 39:227-240, 2003.

R.M. Gous, E.T. Moran, H.R. Stilborn, G.D. Bradford, and G.C. Emmans. Evaluation of the parameters needed to describe the overall growth, the chemical growth, and the growth of feathers and breast muscles of broilers. Poultry Science, 78(6):812-821, 1999.

D. Marquardt. An algorithm for least-squares estimation of nonlinear parameters. SIAM Journal of Applied Mathematics, 11:431-441, 1963.

K.F. Stacey, D.J. Parsons, A.R. Frost, C. Fisher, D. Filmer, and A. Fothergill. An automatic growth and nutrition control system for broiler production. Biosystems Engineering, 89(3):363-371, 2004.

C.M. Wathes, H.H. Kristensen, J.-M. Aerts, and D. Berckmans. Is precision livestock farming an engineer's daydream or nightmare, an animal's friend or foe, and a farmer's panacea or pitfall? Computers and electronics in agriculture, 64(1):2-10, 2008. 